\title{
Should health workers confront patients who they suspect of abusing their partners?
}

\author{
- Cite as: CMAJ 2020 August 17;192:E965-6. doi: 10.1503/cmaj.1095889
}

Posted on cmajnews.com on July 31, 2020

$\mathrm{N}$ ew research from the United States suggests that most young men want their doctors to ask if they've been violent in relationships. But Canadian experts caution that intervening with patients who are abusive may endanger their partners.

A study of 916 American men aged 18 to 35 by researchers at the University of Wisconsin-Madison found that $90 \%$ believed that doctors should ask if they had perpetrated intimate partner violence - which the study defined as any degree of physical violence against a spouse or romantic partner. Yet, only $11 \%$ had ever been asked the question.

About one in five respondents had been violent with a partner, while more than a quarter had survived abuse themselves. These two groups overlapped - most respondents involved in intimate partner violence identified as both a perpetrator and a survivor. Notably, $84 \%$ of men who were violent with a partner still believed doctors should question them.

According to study lead Tova Walsh, "it's really surprising how many men think their doctors should ask them about domestic violence. My hope is that spreading the word about that would lead clinicians to feel more comfortable in asking."

Although there is growing recognition in health care that clinicians have a responsibility to address intimate partner violence, most interventions have focused on survivors, and many clinicians remain hesitant to intervene.

Dr. Elise Wright, a psychiatrist at Women's College Hospital in Toronto, said the reasons physicians do not probe further when they suspect domestic violence are myriad.
"Providers cite issues such as their own personal discomfort, a perceived inadequate knowledge base, forgetting to routinely ask, fears of offending patients by questioning, and a lack of patient privacy," Wright explained at a recent webinar for health workers organized by the University of Toronto. "A lack of appropriate knowledge of referral pathways in the event of a positive screen has also been cited."

The situation is even more complicated when it comes to intervening with patients who perpetrate abuse.

"I do think it would be helpful to speak with them," Wright said in response to a question following the webinar. However, "there is a limited evidence base for things like programs to help men [who are most often the perpetrators] to manage anger, and for things like couples counselling."

Wright also noted that it's unclear if men believing that doctors should ask about domestic violence "speaks to the fact that they may be willing to engage in some sort of treatment."

Michelle Bobala, a registered nurse at Women's College Hospital's sexual assault and domestic violence centre, cautioned that intervening with people who perpetrate abuse could also put their partners at risk of retaliation.

"If they have the same [health care] provider, and they get asked [about domestic violence], and they think they have been targeted, you would worry that they would suspect the victim had

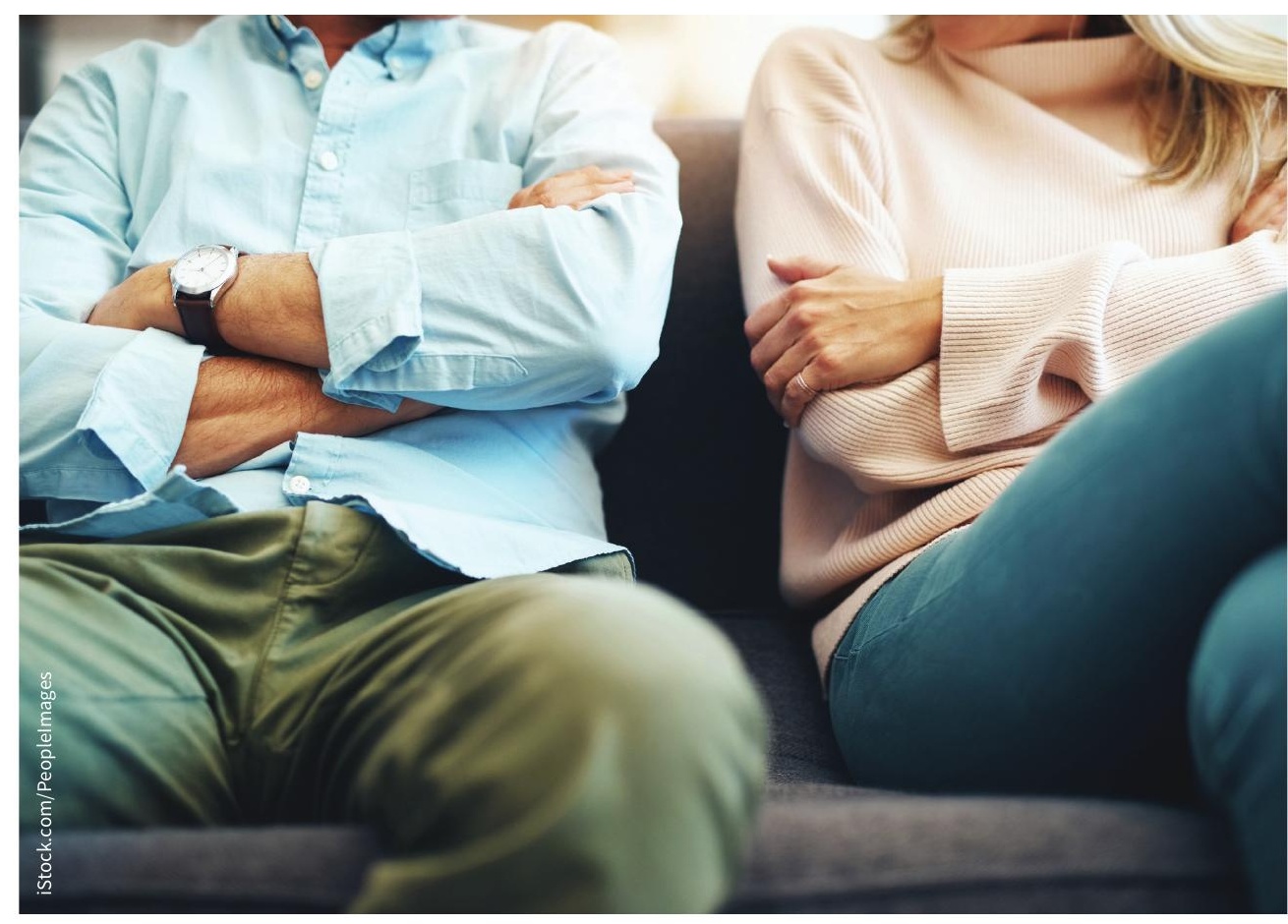

Clinicians are often hesitant to address intimate partner violence. 
disclosed something," Bobala said. "You should really exercise caution."

Futures Without Violence, an American violence prevention group, advises clinicians to speak with patients one-on-one, emphasize the routine nature of questions about domestic violence, avoid blaming language, and use a calm and direct approach when addressing abuse. The group also suggests a variety of interventions, including recommending a trial separation, referral to domestic violence or substance use programs, and advising patients to remove weapons from their homes.

Tensions rising during the pandemic Questions about when and how to intervene in intimate partner violence have become more pressing during the coronavirus disease 2019 (COVID-19) pandemic.

According to Wright, there are concerns that intimate partner violence is on the rise due to couples and families being isolated at home, reduced access to social supports, and economic strain from job losses.

Canadian violence-prevention groups have reported high volumes of calls and website traffic, even as provinces have eased lockdown measures. Yet, intimate partner violence may be underreported because people living with abusive partners may not have the privacy to seek help, Wright said.

Bobala noted that survivors of domestic violence are often escorted by their partner or someone else if they seek medical attention. She suggested that clinicians "find a clever way to separate them for a few minutes," for example, by asking the patient to step into another room to take height and weight measurements or a urine sample.

Clinicians who have moved to telemedicine appointments can still ask about suspected abuse, but they must ensure that the patient is alone and in a safe place, Bobala said. "Do not make an inquiry if you see or hear others in the background. Follow up in a discrete manner by text or call another time if you cannot be certain the patient is alone."

Nonverbal signals are helpful in cases where a partner is likely surveilling the patient, Bobala said. During the COVID-19 pandemic, the "Signal for Help" campaign has encouraged survivors of abuse to use a simple hand gesture on video calls to indicate that they need help.

Clinicians should also be prepared to assist patients in their exit strategies. "Be aware of local resources for sexual assault and domestic violence," Bobala said, noting that they may have to call daily for availability at shelters.

Louise Gagnon, Ottawa, Ont. 